\title{
A novel method for endoscopic closure of endoscopic submucosal dissection-induced defects in the colorectum: the closure method with an elastic-rubber ring (CMER)
}

Endoscopic submucosal dissection (ESD) is a widely used technique associated with high en bloc resection rates for colorectal lesions [1], but at the cost of a higher incidence of adverse events, such as delayed bleeding and perforation, compared with endoscopic mucosal resection [2]. To prevent such complications, closure of ESD-induced mucosal defects is pursued, although this task can become challenging owing to their large size [3]. Herein, we report a new closure method for ESD-induced defects using endoclips and an elastic-rubber ring designed for orthodontics, which we have called the closure method with an elastic-rubber ring (CMER).

CMER is a modification of the loop-clip closure technique using a rubber band $[4,5]$. A 3-mm elastic-rubber ring (Ormco, Orange, California, USA) ( Fig. 1 a) is tied with a 3-0 nylon thread onto either "arm" of a semi-open endoclip (HX-610090; Olympus, Tokyo, Japan) (\Fig. 1 b), which is then retracted into the sheath. The sheath is introduced through a single-channel endoscope and the clip with the attached elastic-rubber ring is "anchored" at the anal side of the ESD defect. The elastic-rubber ring is then "hooked" with another endoclip and fixed to the oral side of the defect. The tension generated by the elastic-rubber ring pulls the resection margins closer. This process is repeated, subsequently leading to further margin approximation and finally to complete closure with the addition of some further endoclips (> Fig. 2).

In the illustrated case, CMER was performed, as described above, post-ESD for cancer in the upper rectum. At repeat endoscopy on day 4 post-ESD, the defect remained completely closed, and complete healing of the defect was documented after 2 months ( $>$ Fig. 3 ; $>$ Video 1 ).

CMER uses tension induced by an elasticrubber ring to reduce the defect size, which thereby facilitates complete clip

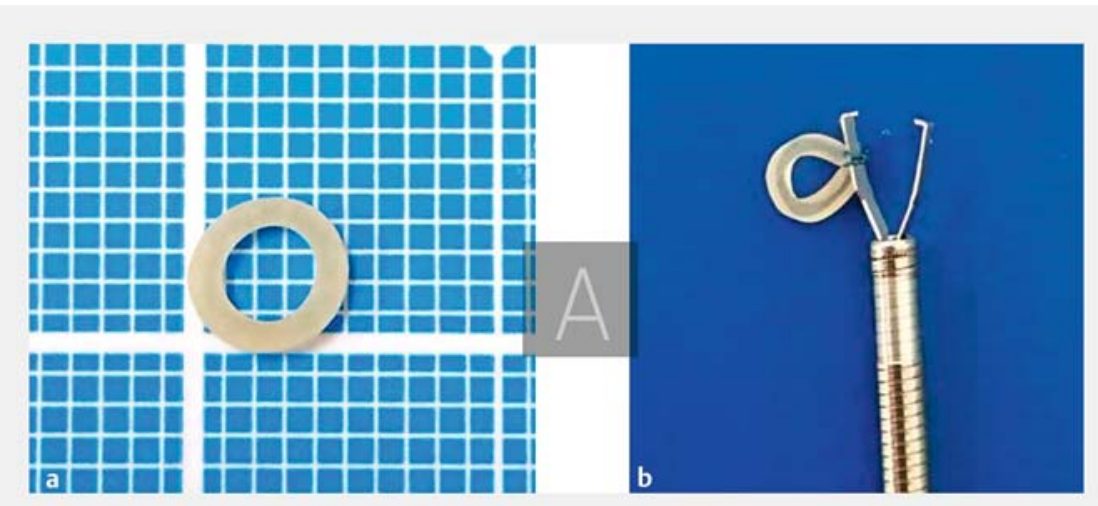

Fig. 1 Photographs showing: a the 3-mm elastic-rubber ring designed for orthodontics; b the rubber ring attached to an endoclip.

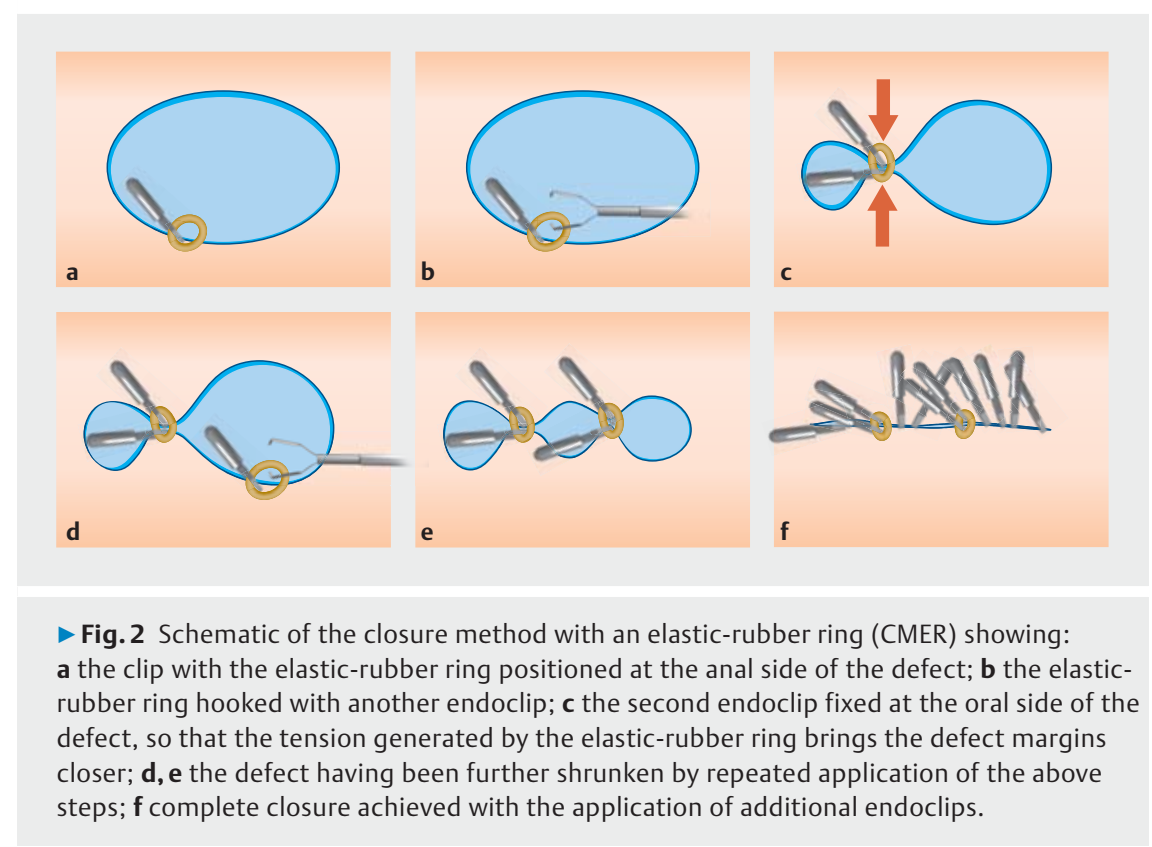

closure, while at the same time distributEndoscopy_UCTN_Code_CCL_1AD_2AB ing the tension burden across the clips to prevent separation. All of the instruments used can be introduced through the working channel, therefore scope retraction and re-insertion is unnecessary. In conclusion, CMER is a simple, inexpensive, and effective technique for complete durable closure of ESD-induced defects in the colorectum. 

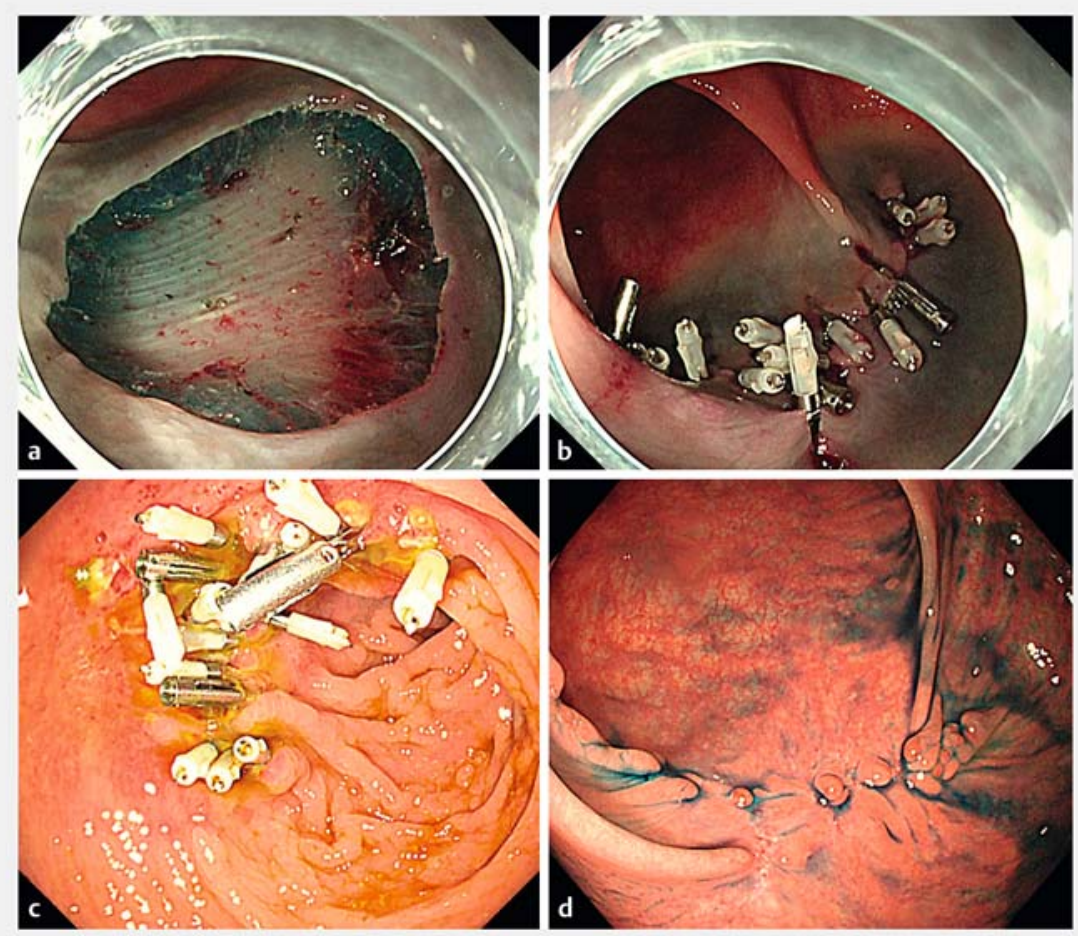

- Fig. 3 Endoscopic images showing: a the mucosal defect after endoscopic submucosal dissection (ESD); $\mathbf{b}$ complete closure of the defect using the closure method with an elasticrubber ring (CMER); $\boldsymbol{c}$ the appearance 4 days post-ESD, with all clips still in place and the defect remaining completely closed; $\mathbf{d}$ the appearance 2 months post-ESD, with complete healing of the mucosal defect evident.
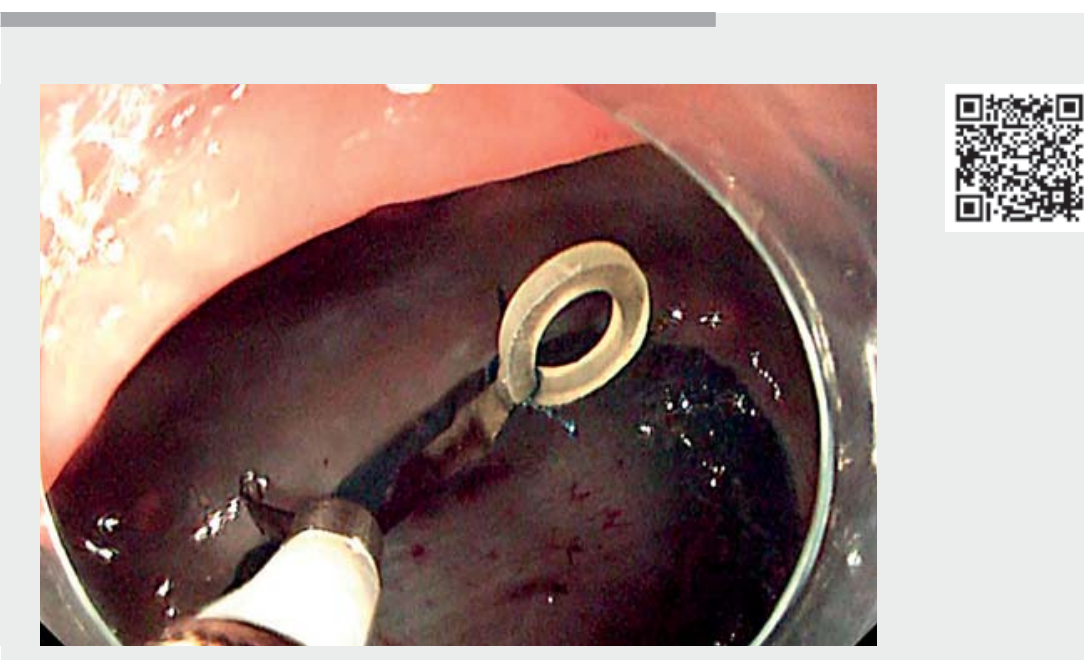

$\checkmark$ Video 1 Application of closure method with an elastic-rubber ring (CMER) to achieve complete closure of a mucosal defect after endoscopic submucosal dissection in the proximal rectum.
The authors

Yuka Kowazaki', Hisashi Fukuda', Itaru Saito' ${ }^{1}$ Toyoaki Sawano ${ }^{2}$, Tomohiro Kurokawa², Norio Kanzaki ${ }^{2}$, Anastasios Manolakis ${ }^{3,4}$

1 Department of Gastroenterology, Jyoban Hospital, Tokiwa Foundation, Fukushima, Japan

2 Department of Surgery, Jyoban Hospital, Tokiwa Foundation, Fukushima, Japan

3 University of Thessaly, School of Medicine, Viopolis-Larissa, Greece

4 Department of Gastroenterology, University Hospital of Larissa, Larissa, Greece

\section{Corresponding author}

\section{Yuka Kowazaki, MD}

Department of Gastroenterology, Jyoban Hospital, Tokiwa Foundation, 57 Jyoban kamiyunagayamachi kaminodai, Iwaki, Fukushima 972-8322, Japan yukaboss_subaru@hotmail.com

\section{References}

[1] Shigita K, Oka S, Tanaka S et al. Long-term outcomes after endoscopic submucosal dissection for superficial colorectal tumors. Gastrointest Endosc 2017; 85: 546-553

[2] Fujiya M, Tanaka K, Dokoshi T et al. Efficacy and adverse events of EMR and endoscopic submucosal dissection for the treatment of colon neoplasms: a meta-analysis of studies comparing EMR and endoscopic submucosal dissection. Gastrointest Endosc 2015; 81: 583-595

[3] Liu M, Zhang $Y$, Wang $Y$ et al. Effect of prophylactic closure on adverse events after colorectal endoscopic submucosal dissection: A meta-analysis. J Gastroenterol Hepatol 2020; 35: 1869-1877

[4] Sakamoto N, Beppu K, Matsumoto K et al. "Loop Clip", a new closure device for large mucosal defects after EMR and ESD. Endoscopy 2008; 40: E97-E98

[5] Lupu A, Jacques J, Rivory J et al. Closure of a mucosal defect with clips and rubber band: a technical trick to improve edge apposition in large mucosal defects. Endoscopy 2018; 50: 726-727

Bibliography

Endoscopy 2022; 54: E407-E408

DOI $10.1055 / \mathrm{a}-1550-2246$

ISSN 0013-726X

published online 8.9.2021

(C) 2021. Thieme. All rights reserved.

Georg Thieme Verlag KG, Rüdigerstraße 14,

70469 Stuttgart, Germany 